\title{
PAKISTAN'S NATIONAL AVIATION POLICY (2015) FLIGHT PATH, CROSS WINDS \& OFF-COURSE
}

\author{
Wali Mughn'1
}

\begin{abstract}
Pakistan is an emerging economy where the aviation policy, promulgated in April 2015, was designed to dramatically boost aviation activities, which in turn was expected to enhance the country's economy. Ownership and market access liberalization, stringent adherence to international standards, subsidies, taxes and duty exemptions/reduction, emphasis on education, investor friendly environment, greater safety and security assurance, and above all, travel and business friendly culture was the strategic direction that Pakistan's forward looking National Aviation Policy anticipated to achieve. Well after a year of promulgation, poor internal and external stakeholder buy-in of the policy continues to mar expectations of the industry's stability, growth and prosperity. This paper critically looks at stakeholder apprehensions and suggests possible remedial measures that may be adopted for a course correction.
\end{abstract}

\section{KEYWORDS}

Aviation policy; Pakistan; liberalization; stakeholder buy-in.

\footnotetext{
${ }^{1}$ Dean \& Director Institute of Business Administration \& Aviation Sciences, 88 DOHS, Phase 2, Malir Cantt, Karachi, Pakistan.

Phone: +923218979014, Email: wmughni@gmail.com
} 


\section{INTRODUCTION}

Pakistan's National Aviation Policy 2015 (NAP-2015), was formulated after 15 years. The policy marked an important milestone in Pakistan's aviation history, where Pakistan's Government along with all stakeholders, representing various segments of the aviation industry, collectively developed a comprehensive and forward looking document. The policy outline and key strategic aspects were also deliberated at length by industry experts and internationally acclaimed aviation policy consultants. While the key objective set forth for aviation division was to develop a safe, secure and efficient air transportation structure, the policy also extended its realm to create an environment to foster economic activity through dynamic and innovative strategic measures. This paper scrutinizes and analyzes overt as well as subtle responses of internal and external stakeholders with regards to policy buy-in. Notwithstanding extensive involvement of stakeholders prior to the formulation of the policy document, the policy once promulgated created dissents amongst many stakeholders. This resulted in widespread criticism of the policy; and the prevalent psyche of operators as well as regulators has de facto formed a barrier for new-entrants and induced greater hindrances for the incumbent operators. The desired cultural change in the existing environment did not come about as the policy failed to effectively bring in the expected values (by incorporating dynamic and bold strategic measures) that the policy hoped to inculcate. With known bugs and anomalies in the previous policy of year 2000, the 2015 policy aimed to rectify and debug weak-links in the system. The Nation Aviation Policy 2015 formulation was initiated with the realization that a non-user-friendly environment existed and that it was pervasively hampering growth and prosperity.

The paper scrutinizes and analyzes the policy's key features and the proposed changes that it aimed to bring about. The study recognizes the positive aspects and then reviews the extent of effective implementation, the degree of stakeholder buy-in and new operational glitches that emerged as a consequence of the new policy. Analyzing the ambiguities and areas that were not readily accepted (and not implemented or half-heartedly implemented), the study further evaluates possible reasons of resistance to change, ingrained cultural inertia and reconciliation with status quo as the preferred option. Concurrently, it was observed that amongst operators in particular there is widespread criticism about the policy that continues to foster an undesirable operational environment that is adversely impacting growth prognosis.

It was also observed that incumbent aviation businesses are eager to grow and expand as the market demand for aviation continues to grow at a fast pace. It is evident that there is a widening gap between supply and demand of various services within the aviation industry. Therefore, while many 
entrepreneurs are enthused to expand their businesses, many are lined up to avail the business opportunities that exists within the aviation space. Notwithstanding the fact that the policy intended to bridge this gap, it unwittingly failed to achieve its major objective of facilitating growth. De facto, many aspects of the policy, the attitude of a number of regulators, and the socio-political environment, are an apparent deterrence to prosperity and growth and subtly but surely the overall milieu is perceived by many as a show-stopper. Such cognitive dissonance to invest or hold (wait and see) continues to bother both incumbent operators and prospective new businesses (market entrants) into the aviation domain.

\section{METHODOLOGY}

To better grasp the on-ground reality and deeper understanding of stakeholder issues, perceptions, popular understanding and common viewpoints about the policy, subjective data and relevant information was gathered through aviation industry grapevine. Prominent aspects were then identified, which were followed by surveys, targeting a diversified sample of stakeholders. Interviews were also conducted, with discussions and deliberations on applicable identified topics, and finally, detailed analysis was done using Q-methodology and factor analysis where applicable.

\section{STUDY OBJECTIVE}

Having realized the prevalent situation and understanding that the intent of the policy formulators was to achieve certain goals through policy implementation; and recognizing the evident diagnosis that the ensuing post policy results have fallen short of the expectations, this paper ventures to recommend and suggest a few remedial measures or corrective actions. To draw an analogy (in the aviation lingo), it may be described as 'a policy that did not fly the expected route', while the cross-winds (criticism and inadequate bury-in by the operators and regulators) continues to drift the industry towards the proverbial uncharted territory of possible downdrafts and wind shears ahead. Finally, moving forward, the paper recommends a course correction to regain the desired track, that the policy originally intended to fly (attain).

\section{IMPLEMENTATION / POLICY BUY-IN: ON-COURSE \& OFF-COURSE}

Hitherto, the service provider function and regulatory function of both Pakistan Civil Aviation Authority (PCAA) and Airports Security Force (ASF) were under their own domains, and this conflict of interest resulted in obvious inefficiencies and latent irregularities. In other words, both ASF and PCAA were service providers and supposedly, regulators/auditors of themselves. Therefore, in order to ensure effectiveness and oversight of aviation safety and 
security in particular, regulatory and service provider functions were made independent of each other with independent functions. According to the policy, Pakistan Civil Aviation Authority shall be the regulator and the Airports Security Force is mandated to be the service provider. Therefore, the inherent conflict of interest is resolved.

Similarly, Safety Investigation Board (SIB), which was under Director General PCAA, is now made independent and now reports to the Minister of Aviation to ensure that findings and safety recommendations of an investigation remain unbiased.

While the above two policy changes were accepted and implemented with zero or negligible resistance, few other aspects that may be considered as continuing bone of contention where reluctance to implementation in true letter and spirit has been observed to be evident. These are discussed in the succeeding paragraphs.

Over the years, protectionism and restrictive market access policy has suppressed the growth potential of the aviation sector in Pakistan. Therefore, transition to more liberal Air Service Agreements (ASAs), was stated in the policy, and it was expected that this would accord greater business freedom, higher levels of customer satisfaction and greater micro and macro-economic growth of the aviation domain (Dawna Rhoades, et al, 2015). As per policy, following the norms and conventions, Pakistan is expected to pursue bilateral open skies policy towards other countries based on the principle of reciprocity.

The proponents of the dissent of this aspect of policy (i.e. liberalization with regards to ASAs) was propagated from executive and managerial level individuals who hailed from the national air careers. Their point of view was projected in a manner that indicated a strong culture of protectionism, and propagating an argument that such a liberal approach was anti-patriotic and that national airline's interest would be compromised. Therefore, a large majority of individuals who subscribe to restrictive market access vehemently opposed the policy in favour of the protectionist approach in the larger interest of the national careers. To ascertain this approach a survey was carried out. The survey results on a Likert scale of 5 indicated a mean of 4.7 with all 20 sample subjects (heterogeneous aviation/airline professionals) specifying their opinion between 4 and 5 (out of 5 ) in favor of dissent to this aspect of policy.

Those policy changes, as stated in the NAP 2015, that appear to be attractive incentives but have not yielded any significant results, except a few half-hearted prospective investors, are discussed below: 
Global trend in participative and cooperative business structures has given credence to the concept of Public-Private Partnership (PPP) models for operation and management of airports. While the private sector specializing in airport management enhances passengers' travel experience by investing in modernization of facilities; the Government is, as per policy, required to focus on the oversight of the operations and ensure accountability of the services, while the private party is expected to run the operations and provide services. PCAA shall (according to the policy guidelines) work with global airport management companies to find suitable PPP models to be followed for the operations and management of landside and terminal facilities of airports. Accordingly, this policy shall also be implemented for small, medium and large airports, with a view to exploit their commercial and tourism potential.

As per the policy, airport infrastructure was to be modernized to meet future needs of aircraft, passenger and cargo traffic. It included refurbishment of airport buildings and rehabilitation of airside infrastructure. Air cargo import and export would further strengthen the business community and help in promoting Pakistani products globally. According to the policy, two state-of-the-art cargo villages are to be established, one in the North and one in the South.

In spite of the incentives offered in the PPP model, companies have not yet come forth to avail the opportunity. It appears that either the policy (and the opportunity) has not been advertised, or not advertised enough, or the incentive package needs to be re-evaluated. In either case, more research needs to be done to identify the cause of poor response.

Aspects of the policy which are grossly misinterpreted are discussed below:

Another impediment in the growth of air travel and cargo was unjustified taxes and duties, which was hampering investment and not yielding any significant revenue for the Government. Rationalization of duties and taxes in the aviation sector shall now (according the stated policy) help attract more businesses, thus resulting in the growth of the industry and ensuing benefits to the end-users. In accordance with the policy, the taxes were supposed to have been restructured and simplified in line with the best international practices. Higher taxes and duties on aviation businesses negatively impact transportation activity in the country, which in turn, adversely impact Gross Domestic Product (GDP) and employment. Therefore, the policy strongly advocates that there should be no taxes and duties on investment in aviation sector. 
The objective and the intent of this policy regarding tax breaks and exemption of CAA charges was to facilitate incumbent operators and to attract investors in various sectors of aviation business, including establishment of quality Maintenance, Repair \& Overhaul (MRO) organizations.

Notwithstanding the above, the statement of the policy (para 4.8 [b]) guideline that "Import or lease (wet/damp/dry) of any General Aviation (GA) aircraft shall be tax and duty free", was misinterpreted and the commercial aircraft category selected was any aircraft that weighed above 15,000 kg was put under Pakistan Customs Tax Free regime. Those aircraft that were below this weight limit were not mentioned, and therefore, not included. Pakistan Customs allots Pakistan Customs Tariff codes (called PCT codes) and publishes all leviable duties and taxes against them. The PCT code allotted for exemption of duties and taxes is 8802.4000 , which is for all categories of aircraft weighing above $15,000 \mathrm{~kg}$. It is also worth noting that that EASA (European Aviation Safety Agency) refers to the Convention on International Civil Aviation, and quotes its Annex 8 (for Airworthiness of Aircraft), that specifies standards that fixed wing aircraft with maximum takeoff weight of greater than $5,700 \mathrm{~kg}$ must comply with. Furthermore, most General Aviation (GA) aircraft are below $5,700 \mathrm{~kg}$ MTOW (Maximum Takeoff Weight) category. Therefore, this misinterpretation appears to be arbitrary and not based on any weight category that could be confused or misinterpreted with. The only place where $15,000 \mathrm{~kg}$ (MTOW) category of aircraft appears is airport landing and parking fee structure of various airports across the globe. It can thus be assumed that $15,000 \mathrm{~kg}$ limit is not based upon any precedence or relevant logic.

Similarly, the policy envisaged reduction and/or removal of taxes and duties on GA aircraft, aircraft spares and material for maintenance of GA aircraft are also required to be given the same status. The policy did not get implemented in letter and spirit; additionally, it also introduced more complications in the process of import which should have been made simpler and user friendly. Thus, cumbersome and time consuming processes are negating the intent and purpose of the policy. Procedural complexities and misinterpretation in this area of operation is hurting the GA operations in particular and the aviation industry in general. This information was given by few senior GA operators and the case was further investigated and found to be absolutely true and valid.

The policy guideline to address the 100 LL (Low Lead) supply and distribution monopoly has also proven ineffective. No change to monopolistic fuel supplier for aviation fuel for GA operations i.e. $100 \mathrm{LL}$ continues to plague GA operations, particularly the flight training 
operation. Albeit it is very clearly and categorically mentioned in the policy that the monopoly of supplier and distributor of $100 \mathrm{LL}$ aviation fuel shall be addressed so that cost of fuel is reduced (due fair competition), nothing concrete is visible to break the monopolistic powers of the supplier. It is known that fuel cost can be as high as $75 \%$ of the Variable Cost of GA operations. Therefore, to contain costs and offer flying training at an affordable price, it is incumbent on the Government to reduce the price of fuel. It can be done by incentivizing new entrants (suppliers of $100 \mathrm{LL}$ ) and breaking the monopolistic approach of a single supplier. Alternatively, a cost cap was also not enforced, that could have kept the cap (upper limit) to preclude exorbitant prices. Resultantly the GA operation continues to suffer, and the end result is that the training of pilots has become cost prohibitive to many prospects who can ill-afford the fees that the flight training schools are charging to sustain their operations.

Recent air crashes and incidents have highlighted the need for stringent checks and procedures. In accordance with the new policy, PCAA is now reviewing such procedures and regulations for greater safety and efficiency of air transportation services. The policy also encourages induction and operation of more efficient aircraft by Pakistani operators. Such modern aircraft are safer and more fuel efficient. As a measure to ensure that only sound investors/operators venture in the aviation business, the paid-up capital requirement to obtain relevant licenses has been enhanced and the chronological age of aircraft for initial induction has been capped at 12 years.

The chronological age limitation to induct aircraft less than 12 years was introduced for the following reasons and logical assumptions:

a) Twelve-year period was primarily based on two ' $D$ ' checks which are required to be completed on aircraft (which are generally approximated at 6 years' chronological age interval).

b) Most newer aircraft ( $\leq 12$ years' chronological age) are relatively more fuel efficient than their older counterparts.

c) Most newer aircraft ( $\leq 12$ years' chronological age) are relatively greener than older aircraft.

d) Most newer aircraft ( $\leq 12$ years' chronological age) have better navigation and safety equipment installed as compared with their older versions.

e) The maintenance cost of newer aircraft ( $\leq 12$ years' chronological age) is generally less than older counterparts. 
f) This also indicates that the aviation business entity that is buying or leasing the aircraft are financially stronger than those who look for relatively older and cheaper aircraft.

g) While there are research studies that have indicated that there is no significant correlation between age of aircraft and fatal accidents up to 26 years of chronological age (Hansman 2012), there are a few studies that suggest that there is a positive correlation (Vasigh and Herrera, 2009) in their comprehensive study on "A Basic Analysis of Aging Aircraft, Region of The World, And Accidents." Interestingly, the rate of accidents and the probability of incidents, occurrences and accidents drop significantly just after 6 years (i.e. after ' $D$ ' Check, see Figure 1 below). The study was conducted on a global database of 549 aircraft accidents analyzed between 2000 and 2007.

Figure 1: Number of Accidents

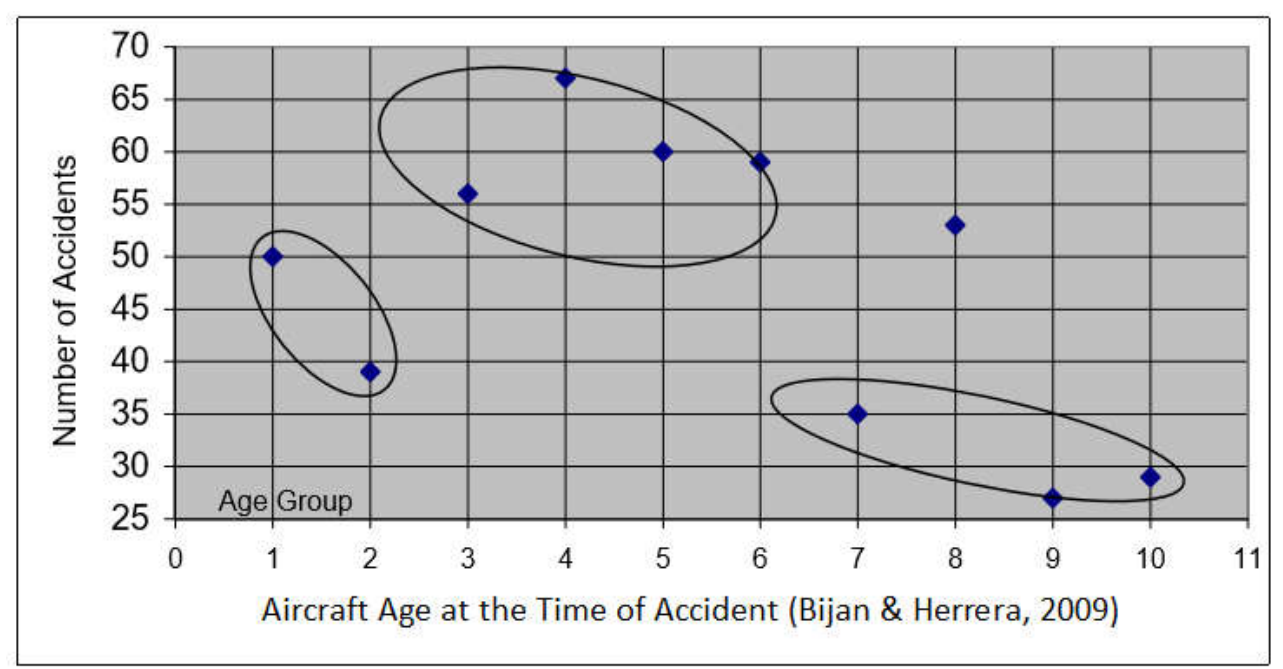

h) With reference to the study stated above, it appears that statistically and historically, the safest period in the life of an aircraft is just after ' $D$ ' Check. Thus, when the policy states less than 12 years it is assumed that businesses desirous of inducting aircraft for RPT (Regular Public Transport) operations would like to induct aircraft just after the first ' $D$ ' Check. Induction of such aircraft would have the highest probability of safety (least accident probability).

Table 1: Aircraft Accidents by Region

\begin{tabular}{|ccc|}
\hline \multicolumn{3}{|c|}{ Aircraft Accidents by Region } \\
& Accidents & Probability \\
& 121 & $25.42 \%$ \\
North America (NA) & 60 & $12.61 \%$ \\
Latin America (LA) & 99 & $20.80 \%$ \\
Europe (EU) & 69 & $14.50 \%$ \\
Africa/Middle East (AF) & 127 & $26.68 \%$ \\
Asia/Asia Pacific (AP) & 476 & $100.00 \%$ \\
Total & Source: Vasigh \& Herrera 2009 \\
\hline \multicolumn{2}{|c}{} \\
\hline
\end{tabular}


i) Another study (Vasigh \& Herrera 2009, see Table 1 above) also clearly indicates that Pakistan falls in a region where the probability of accidents is one of the highest in world. Therefore, to mitigate other factors and reduce the probability of occurrence, all possible measures must be undertaken for ensuring greater safety. Therefore, it can be concluded that 12-year chronological age limitation for induction of aircraft is a positive step and must be taken in the right spirit.

Another concern sounded by most individuals is an objection to the enhanced paid-up capital limit. For domestic charter operations, the new policy requires that a company applying for the domestic charter license would be required to show a paid-up capital of Rs. 25 million. Although this seems like an excessive hike in the required amount (from previously 2 million to the new limit that is 12.5 times higher); it may be noted that the amount approximates only $\$ 250,000$. A company that desires to enter the aviation business offering charter services must at least be able to bear the monthly lease charges, maintenance charges and insurance charges and that may add up and exceed the paid-up capital stated in the policy. In the larger interest of the public that a new entrant wants to serve the market, it must have enough financial strength to sustain initial operations and bear losses as may be expected during the initial period after commencing operations.

Wet lease cost will be no less for aircraft typically deployed in an RPT operation. The required paid-up capital for RPT license is only $\$ 5,000,000$ (approx.) which for an operation with at least 3 aircraft (another policy requirement) would not be enough to cushion losses before break-even level is attained. Since the public is involved (as customers and clientele who may plan their business and travel requirements with the growth of the aviation services in the region, may be adversely affected if an airline closes down, bankrupts or simply performs inefficiently or compromises safety. It may be noted that there is a recent history of failures of new entrants because of lack of adequate financial strength, wherein the concerned entrepreneurs suffered losses while the general public was deprived of the services promised to them. Due to such repeated failures due to insufficient funding capability of the RPT operators, the aviation business environment has suffered in terms of consumer confidence, reputation and safety record. Therefore, it may be argued that it is reasonable to expect better performance by a more serious and financially sound new entrant in the aviation business. Thus the policy justifies the economic oversight as required by ICAO.

Scheduled routes to politically and socially deprived locations are now ear-marked to be served for those entrepreneurial business entities who want to avail the opportunity. According to 
the policy, the operators are offered attractive incentives such as waived-off fees and other allied charges while operating to and from these locations. For such operations a new term was coined -- 'socio-political' routes. These routes are planned because political integration and social welfare was the primary objective of this operation and economic considerations were secondary.

Notwithstanding the incentives, there has been little or no response from the entrepreneurs to avail this business opportunity. The reason for such a lackluster response could be as follows:

a) Lack of awareness about the opportunity, particularly for those who are capable of undertaking this venture.

b) Not enough conducive environment for the entrepreneurs to venture in to such business.

c) Non-business friendly attitude of the PCAA (Pakistan Civil Aviation Authority). This aspect is based on a few random interviews with entrepreneurs considering market entry in the aviation domain. A survey is underway to ascertain reasons with a greater degree of confidence.

d) Perceived insecure environment (security concerns) in the remote areas where the identified airfields are located on the specified 'socio-political' routes.

The policy puts strong emphasis on up-gradation of air navigation infrastructure and effective utilization of satellite based technology to improve safety standards and future capacity needs of air traffic. However, except a few positive changes, not much improvement in the facilities is visible and it appears that the priorities are moving away from what was expected from the policy.

In order to capitalize the true potential of General Aviation (GA), apart from routine training of aviation personnel, other GA areas like aero-sports, tourism, agricultural pesticide and seeding sprays, cloud seeding, etc. would be encouraged and facilitated wherever considered possible and appropriate. Befitting incentives were to be offered for the growth of this sector. This seems to be an unattended and unaddressed area of the stipulated GA growth plans indicated in the policy.

Keeping in view the rapidly changing technology in the aviation industry, training and skill development of aviation personnel has also been given due importance in this policy, but no effort by the authorities to promote training and education is visible. This is a perception 
shared by most incumbent aviation training and education institutes. This too is a survey based conclusion.

\section{IMPEDIMENTS TO SUSTAINED GROWTH (DRAG)}

Issues that are perceived as road blocks to good governance, development and sustained growth of the industry were identified and are summarized below:

There has been inadequate human resource development. The regulators as well as the service providers lack adequate formal training on international standards. The operators too short handed on qualified human resource with desired training, certifications and educational qualifications. Training and education in all segments of aviation industry must be given serious attention and priority. Formal training of technicians, education in aviation management for managers, international certifications for air traffic controllers, air transport \& economic regulators, airspace and aerodrome safety regulators, airworthiness and flight standard inspectors are few of the areas that deserve attention. Emphasis on human resource development in finance and information technology is also lacking. Additionally, there seems to be a perpetual shortage of regulators, particularly flight standards and airworthiness regulators. This shortage adversely affects ramp inspections which the industry needs for efficient approvals and certification processes.

The policy emphasizes that there is a cultural change required in being customer-centric, safety and security-conscious and being positive in our attitude with high moral values and good work ethics for all PCAA personnel in particular and the aviation industry in general. This is a known weakness and concerted efforts need to be initiated to bring about a constructive change. Changing culture takes time and results will not be immediate, but we must initiate the efforts to make the desired change today. The future of Pakistan's aviation system depends on this change.

There is slow and inadequate infrastructure development (aerodrome facilities and navigation facilities on the airside). This aspect has been neglected in the past and is supposed to be addressed on priority. There are also huge gaps in communication and surveillance coverage in the western and northern part of Pakistan airspace due to limitations of conventional transmitters and sensors in hilly terrain. This could have been resolved long ago by application of satellite technology like CPDLC (Controller Pilot Data Link Communication), and ADS(C) [Automatic Dependent Surveillance (Contract)]. These technologies are ideally suited not only for the hilly terrain but are also cheaper alternates to conventional tools of 
communication and surveillance necessary for air traffic control at long distances. Hence, action to aggressively address this issue is required as it relates to safety of air traffic transiting through Pakistan airspace.

The "procedures and regulations" are not user-friendly and act as a barrier to entry for new entrants in the industry. This aspect also needs to be addressed as soon as possible.

There is inadequate technology awareness, adoption and usage in all segments of the industry.

There is insufficient commercialization of non-aeronautical areas at the airports (e.g., real estate, car parking, food \& beverages, retail stores, hotels, commercial plazas, etc.)

In the past, National Aviation Policy was also not implemented effectively. Therefore, National Aviation Policy-2015 implementation plan forms an integral component of the policy and timely implementation and follow up are structured in the system. Progress audit and remedial measures for non-performance is to be enforced. Steps to ensure implementation, stakeholder buy-in, and continual evaluation and assessment to see if the industry is moving on-track and on-schedule is important and critical to success.

\section{RECOMMENDATIONS \& CONCLUSION (COURSE CORRECTION CHECK-LIST)}

It is conclusively determined that the buy-in of policy is equally important for successful implementation of the policy. In retrospect, it is agreed by most policy contributors (consultants, regulators and operators) that before the next policy is formally approved it is recommended that the policy's various aspects may be circulated and formal and informal feedback must be taken and evaluated in a timely manner, and more seriously and comprehensively than it was done before. Furthermore, road shows, seminars and conferences may be conducted so as to inform and educate the stakeholders. Once the policy is formulated and approved, it must be implemented effectively. Periodically and in a systematic manner, the achievements must be measured, and time-lines considered, while never losing sight of the desired objectives of the policy.

The results of the policy must favorably impact local, regional and global business entities and passengers travelling within, as well as to-and-from Pakistan.

Incentivized involvement of foreign investors is a key component of the policy and incentive packages may be re-evaluated and tweaked (wherever necessary) to get better results. The 
involvement of international reputable organizations and institutions is certainly expected to bring about greater collaboration, a favorable cultural mix and much needed enhanced confidence amongst all national and international participants. Economic well-being for all industry participants and stakeholders is a collateral advantage of the measures envisioned in the policy.

Periodic evaluations, change management and buy-in efforts through print, electronic and social media must also be exploited.

Reasons of resistance to change also need to be studied and evaluated. Deliberated corrective measures will have to be continually implemented and monitored to bring about a positive cultural change.

Table 2

\begin{tabular}{|l|l|l|}
\hline \multicolumn{3}{|c|}{ Strategy to Cater for Resistance to Change } \\
\hline \multicolumn{1}{|c|}{ Approach } & \multicolumn{1}{|c|}{ Situation } & \multicolumn{1}{c|}{ Advantages } \\
\hline $\begin{array}{l}\text { Education }+ \\
\text { Communication }\end{array}$ & $\begin{array}{l}\text { Where there is lack of } \\
\text { information or inaccurate } \\
\text { information and analysis }\end{array}$ & $\begin{array}{l}\text { Once persuaded, people will often help } \\
\text { with the implementation of the change }\end{array}$ \\
\hline $\begin{array}{l}\text { Participation }+ \\
\text { Involvement }\end{array}$ & $\begin{array}{l}\text { When a change in design is } \\
\text { necessitated and there is } \\
\text { considerable power to resist }\end{array}$ & $\begin{array}{l}\text { People who participate will be } \\
\text { committed to implement change and } \\
\text { relevant inforamtion will be integrated } \\
\text { in the change plan }\end{array}$ \\
\hline
\end{tabular}

Source: Harvard Business Review July-Aug 2008, Kotter \& Schlesinger

"It must be considered that there is nothing more difficult to carry out, nor more doubtful of success, nor more dangerous to handle, than to initiate a new order of things." Niccolò Machiavelli, The Prince.

As shown in Table 2 above, the strategy to cater for the resistance to change is primarily through apt education and effective communication. This strategy as elaborated and discussed by Kotter \& Schlesinger's article published in Harvard Business Review magazine (July 2008) is considered most suitable where there is a general lack of complete knowledge or inaccurate information about the subject at hand. Inaccurate analysis leads to false beliefs, and commonly accepted norms are difficult to change.

As aptly quoted by Kotter \& Schlesinger: "It follows that an acceleration in the rate of change will result in an increasing need for reorganization. Reorganization is usually feared, because it means disturbance of the status quo, a threat to people's vested interests in their 
jobs, and an upset to established ways of doing things. For these reasons, needed reorganization is often deferred, with a resulting loss in effectiveness and an increase in costs."

When a policy demands macro level change, macro level change management must follow. It is also pertinent to note the fact that an amenable relationship between the initiators and the people is mission critical for success. Equally important is a sincere effort, logical approach and a few dedicated professionals who may be taken on-board to make a team that disseminates and propagates the concepts that form the essential drivers to instill a change that is pervasive and progressive.

To get back to the desired track, all the above shall have to be considered. Albeit, time is of essence and it takes time to change, the effort must commence today to expect a positive change in the days and months to come.

\section{ACKNOWLEDGEMENTS}

Apart from valuable suggestions and contributions by many stakeholders from the aviation industry, including operators and regulators, few critical contributors that were considered quintessential, pertinent and significant came from a consulting team from Embry-Riddle Aeronautical University. They reviewed, guided and helped in navigating the policy formulation process. In this context special appreciation goes to the following: a) Prof. Dr. Dawna L. Rhoades, b) Prof. Dr. Ahmed Abdelghany, c) Prof. Dr. Bijan Vasigh, and d) Prof. Dr. Chunyan Yu. On behalf of Pakistan's aviation industry and the academia, efforts of all contributors, consultants and stakeholders who helped in the study by sharing candid views on post-policy effect, analysis and evaluation of implementation of the policy is also appreciated. Thank you.

\section{REFERENCES}

1. Journal of Air Transport Management 47 (2015) 102-111. Aviation Planning Policy in Australia: Identifying Frames of Reference to Support Public Decision Making. Robert Kivits, Michael B. Charles. Southern Cross University, Bilinga, Australia 
2. Analysis of Impact of Aircraft Age on Safety for Air Transport Jet Airplanes (2012). Professor R. John Hansman from MIT) International Center of Air Transportation, Cambridge, USA.

3. Australian Transport Safety Bureau. (2007). How Old is Too Old? The impact of ageing aircraft on aviation safety. Canberra City, AU: Australian Transport Safety Bureau.

4. Journal of Business \& Economics Research - May, 2009. A Basic Analysis of Aging Aircraft, Regions of the World, and Accidents. Bijan Vasigh \& Jorge Herrera, EmbryRiddle Aeronautical University, USA

5. Boeing. (2008). Boeing: History-Higher, faster, farther- McDonnell Douglas Corp...Goshawks and MDs. Retrieved May 23, 2016, from the Boeing Company: http://www.boeing.com/history/narrative/n085mdc.html

6. Boeing Commercial Airplanes. (2007). Current market outlook 2007: How will you travel through life? Retrieved September12, 2015 from http://www.boeing.com/commercial/cmo/index.html

7. Borenstein, Severin \& Nancy L. Rose. (2007). "How Airline Markets Work...Or Do They? Regulatory Reform in the Airline Industry," NBER Working Papers 13452, National Bureau of Economic Research.

8. Dempsey, P. and L. Gesell. (1997). Air Transportation: Foundations for the 21st Century. Chandler: Coast Aire Publications.

9. Dionne, G., Gagne, R., Gagnon, F., \& Vanasse, C. (1997). Debt, moral hazard and airline safety: an empirical evidence.

10. Journal of Econometrics, 79, 379-402. 8. FAA. (2006). Controlling Fleet Growth and Managing Aircraft. Twentieth Meeting of Directors of Civil Aviation of the Eastern Caribbean, (p. 5).

11. Vasigh, B., and Helmsky, S., "Airline Safety: An Application of Empirical Methods to Determine Fatality." Handbook of Airline Economics, Second Edition, PP 501-512, McGraw- Hill Company, 2002

12. Dick Forsberg, "Aircraft Retirement \& Storage Trends - Economic Life Analysis Reprised and Expanded" (2015)

13. PhD thesis; Pruneddu, A. (2013) Implicit person theories and Q-sort: Personality change in emerging adults. PhD thesis, University of York, Alessio_Pruneddu_PhD_Thesis_December_2013.pdf

14. Aviation Policy Framework (2013). Presentation to the Parliament by the Secretary of State for Transport by Command of Her Majesty. United Kingdom. 
15. Flight Path 2050 (2011). Europe's Vision for Aviation. Report of the High Level Group on Aviation Research. European Union

16. The Economic Impact of Air Transport Industry on National Economy of Pakistan (2014) Dr. Bijan Vasigh, Dr. Dawna Rhodes, Dr. Chunyan Yu, Dr. Ahmed Abdelghany, EmbryRiddle Aeronautical University, Daytona Beach, FL, USA

17. Brown, J.S., Duguid, P. (1996) Organizational Learning and Communities-of-

Practice: Toward a Unified View of Working, Learning, And Innovation, Organizational Learning, Sage Publications, Thousand Oaks 